Harold Bula, Sistema Experto Berenjena

\title{
SISTEMA EXPERTO PARA EL DIAGNÓSTICO DE PLAGAS Y ENFERMEDADES EN LOS CULTIVOS DE BERENJENA (Solanum melongena L.) EN LA REGIÓN CARIBE DE COLOMBIA.
}

\section{EXPERT SYSTEM FOR THE DIAGNOSIS OF PESTS AND DISEASES ON CROPS IN THE REGION OF EGGPLANTCARIBBEAN COLOMBIA.}

\author{
Harold D. Bula ${ }^{1}$, Hermes Aramendiz ${ }^{2}$, Daniel J. Salas ${ }^{3}$, Wilmer E. Vergara ${ }^{4}$, Aida L. Villadiego ${ }^{4}$. \\ Recibido para publicación: 28 de junio de 2012 - Aceptado para publicación: 13 de Octubre de 2012
}

\section{RESUMEN}

El presente trabajo se basó en la construcción de un sistema experto para el diagnóstico de plagas y enfermedades en los cultivos de berenjena en la región Caribe de Colombia, con el objetivo de ponerlo a disposición de los profesionales del agro dedicados a esta labor, y de esta manera facilitar el diagnóstico de plagas y enfermedades en este cultivo. El trabajo se realizó con una base de conocimiento la cual se adquirió mediante la participación de un experto en el área del Departamento de Ingeniería Agronómica y Desarrollo rural de la Universidad de Córdoba. Su construcción combina tecnologías tales como, swi-prolog, JAVA TM, PostgrSQL y XML y fue desarrollado bajo los principios actuales de la Ingeniería del Software y soportado en un enfoque metodológico que garantiza confiabilidad y consistencia del sistema. El sistema experto es capaz de diagnosticar ocho (8) plagas y nueve (9) enfermedades de los cultivos de berenjena, por medio de dos módulos de inferencia, donde se tienen en cuenta los daños presentes en las diferentes partes de la planta, así como la presencia de algún tipo de insecto. Adicionalmente se debe ingresar la estación climática, con el fin de dar un diagnóstico más certero.

PALABRAS CLAVES: Inteligencia Artificial, Sistemas Expertos, SWI-Prolog, cultivos de berenjena, Fitosanidad.

\footnotetext{
${ }^{1}$ Ingeniero Sistemas, MSc. Profesor titular, Universidad de Córdoba, Montería - Colombia habulahe@hotmail.com

2 Ingeniero Agrónomo, Ph.D. Profesor titular, Universidad de Córdoba, Montería

3 Ingeniero Sistemas, MSc. Profesor titular, Universidad de Córdoba, Montería

${ }^{4}$ Ingeniero de sistemas, universidad de córdoba, Montería
} 
Harold Bula, Sistema Experto Berenjena

\section{ABSTRACT}

This work was based on building an expert system to diagnose pests and diseases ineggplant crops in the Caribbean region of Colombia, with the aim of making it available to the agricultural professionals engaged in this task, and thus facilitating the diagnosis of pests and diseases in this crop. The work was done with a knowledge base whichwas acquired through the participation of an expert in the area of the Department of Agricultural Engineering and Rural Development at the University of Cordoba. Its construction combines technologies such as swi-prolog, JAVA TM, PostgreSQL and XML and was developed under the current principles of Software Engineering andsupported on a methodological approach that ensures reliability and consistency of the system. The expert system is able to diagnose eight (8) pests and nine (9) of crop diseases eggplant, by means of two modules of inference, which take into account the damage present in different parts of the plant, and the presence of some kind of insect.Additionally you must enter the weather station, to give a more accurate diagnosis.

Keywords: Artificial Intelligence, Expert Systems, SWI-Prolog, eggplant crops, Crop Protection.

\section{INTRODUCCIÓN}

La Berenjena (Solanum melongena L.) es una especie hortícola originaria del continente asiático, que pertenece a la familia Solanaceae, y es cultivada desde hace más de cuatro milenios, y fue introducida a Colombia en la década 30 del siglo XIX por los inmigrantes Árabes, donde hace parte importante de la gastronomía del Caribe Colombiano. Esta región del país cuenta con una oferta ambiental favorable y una posición geográfica competitiva; pero debido a la falta de tecnología y el debido manejo agronómico, Colombia se encuentra muy por debajo con relación a otros países en cuanto al rendimiento por unidad de superficie, $20 t^{*}$ ha - 1 frente a España $50 t^{*} h a^{-1}$ [1].

A nivel nacional la producción de hortalizas en general y la berenjena en particular, está en manos de pequeños agricultores, en parcelas de poca extensión, manipuladas con insuficiente tecnología, el esquema administrativo es muy tradicionalista es decir, las decisiones se toman en base a la intuición, a la percepción y no se tiene en cuenta la información relevante que permita una buena toma de decisión. Esto se convierte en un obstáculo para el desarrollo agrícola y la competitividad en una economía globalizada, que demanda productos inocuos a la salud del consumidor. Por otro lado no siempre se contará con la ayuda de un profesional del agro que asista a la toma de decisiones y si lo está, es posible que su posición cambie radicalmente frente al mismo conjunto de variables en contextos parecidos.

El sector productivo y la tecnología siempre han ido de la mano, dando como resultado increíbles investigaciones, y han aportado grandes avances al desarrollo de sistemas enfocados a apoyar específicas actividades del sector productivo. La Inteligencia Artificial y una de sus ramas, los sistemas expertos, con un enfoque totalmente diferente al resto de los sistemas existentes, brindan la posibilidad de tomar decisiones de manera más precisa y rápida de lo que un ser humano lo haría, lo que los hace una herramienta potente dado a que está en la capacidad de procesar grandes volúmenes de información, sin correr el riesgo de tomar una decisión errónea, por no tener en cuenta datos que considere innecesarios.

Analizando las ventajas de tener un sistema que asista el diagnóstico de plagas y enfermedades que afecta la Berenjena, y teniendo en cuenta que de esta forma serán tomadas decisiones preventivas y correctivas que ayuden a mejorar la productividad de toda una región.

El objetivo primordial de este estudio fue el diseño e implementación de un sistema 
Harold Bula, Sistema Experto Berenjena

experto, para diagnosticar plagas $y$ enfermedades en los cultivos de berenjena en la región Caribe de Colombia.

\section{ESTADO DEL ARTE}

En el año 1950 el matemático, científico de la computación, criptógrafo y filósofo inglés Alan Turing, publicó un trabajo titulado "Inteligencia y Funcionamiento de las Máquinas" con el fin de demostrar hasta qué punto estas tienen inteligencia. Más tarde en 1965 se empezaron a utilizar técnicas para la resolución de problemas que se caracterizaban por la búsqueda heurística como modelo para la resolución de problemas, y con ellas comenzó la investigación y desarrollo de los sistemas expertos[2].

Después de hacer un estudio detallado de los comienzos de los Sistemas expertos por todo el mudo se encontró que:

En Europa desde el año de 1999 se desarrolló el proyecto SPONGIA, en el centro de estudios avanzados de Blanes (CEAB-CSIC) y en El Instituto de Investigación en I.A. (IIIA-CSIC) de España más específicamente de la ciudad de Cataluña. El proyecto consistió en la creación de un S.E. que permitiera la identificación de esponjas marinas del área atlantomediterránea. SPONGIA, nació de una tesis doctoral llevada a cabo por la investigadora Marta Domingo, quien realizado su tesis en el Instituto de Investigación en I.A. (IIIA-CSIC). El S.E. está capacitado para identificar alrededor de 100 grupos taxonómicos de esponjas marinas concernientes a la clase Demospongiae, a la cual corresponden la mayor parte de las esponjas conocidas. El gran interés para desarrollar un S.E. de identificación de esponjas radico principalmente en la dificultad que normalmente se tiene al momento del reconocimiento de estas. SPONGIA se maneja partiendo de unas reglas de pregunta $y$ respuesta. SPONGIA asesora a través de preguntas al usuario que se encuentre observando la esponja. El usuario puede manifestar incerteza en la respuesta 0 proporcionar otras opciones entre las cuales no se encuentra seguro o, decir que desconoce lo que se le pregunta.
El Instituto de Investigación en I.A. (IIIA) también desarrollado otros S.E. por mencionar en el campo de la medicina (Pneumon-IA y Terap-IA los cuales son sistemas de asistencia para el análisis y el método de neumonías). Por otra parte en el campo de la ganadería creo PORCIA, un S.E. se encarga de ayudar a la gestión de granjas de cerdos. En el 2003 la Escuela Universitaria de Formación del profesorado "Pablo Montesinos" de España (Madrid), llevo a cabo el proyecto Animalandia el cual se constituyó como una técnica libre de primer orden para cualquiera cercanía pedagógica al mundo animal, fuera cual fuera el escalafón educativo. Este proyecto Incluyo la información concerniente a más de 1000 niveles taxonómicos y de más de 2750 especies de animales. Animalandia cuenta con casi 4000 imágenes libres de más de 1200 géneros diferentes y series de vídeo.

En Asia para el año de 1990 se desarrolló un SE para la evaluación de un sistema de apoyo a la toma de decisiones en las etapas previas a cirugía del cáncer de próstata, por EI Departamento de Urología del Hospital Memorial Chang Gung y la Universidad de Chang Gung, Taoyuan, Taiwan. El estudio se realizó con 43 pacientes para previa cirugía de cáncer de próstata, y fue comparado los resultados de cuatro urólogos y cinco residentes de urología arrojando los siguientes resultados $88.4 \%$ de exactitud para la evaluación del sistema de apoyo a la toma de decisiones en las etapas previas a cirugía del cáncer de próstata, superando a dos urólogos y a todos los residentes de urología. El sistema podría ser usado para evitar cirugías innecesarias de cáncer de próstata [3].

En Africa se desarrolló TOMATEX un sistema Experto para tomates, este suministra recomendaciones. $\mathrm{Y}$, trata de investigar los desórdenes más típicos de estas plantas para así dar especificaciones para una solución óptima. Tomatex está basado en dos subsistemas: Subsistema de diagnóstico y Subsistema de Tratamiento. Este sistema fue liderado por Egyptian Central Laboratory for Agricultural Expert Systems CLAES. 
Harold Bula, Sistema Experto Berenjena

En América el proyector DENDRAL se originó desde el año de 1965, después que el señor Feighembaum, ingreso a la Universidad de Stanford, este comenzó a trabajar al lado del profesor Joshua Lederberg, un Químico Molecular especializado. Estos dos investigadores, tenían una misma inquietud de poder usar computadores para el modelamiento del pensamiento científico, es decir, un programa que admitiera enumerar todas las distribuciones viables que se pudieran ajustar a los datos de partida, datos que iban a ser obtenidos del espectrómetro másico, y que permitiera eliminar aquellos que no cumplieran las restricciones propuestas, para así lograr un análisis eficiente de las moléculas complejas, sabiendo que este tipo de dificultades son de complejidad exponencial. Así nació DENDRAL como un S.E. que permite el problema químico planteado anteriormente por los investigadores, mediante un proceso de indagación de creación y de una prueba jerárquica. Su base de conocimientos se desprende de dos conjuntos de reglas que corresponden a cada fase del desarrollo del sistema.

Macsyma es un S.E. de algebra computacional que fue desarrollado originalmente desde 1968 hasta 1982 en el MIT como parte del proyecto MAC, fue uno de los primeros S.E. basados en conocimiento y el primero en matemática simbólica global. Más adelante surgio Mycin un S.E. que empezó su desarrollado en principio de los años 70 por Edgar ShortLiffe, investigador de la Universidad de Stanford. MYCIN fue escrito en el lenguaje Lips e inspirado en sus comienzos en DENDRAL. Su primordial ocupación permanecía en la determinación de enfermedades infecciosas de la sangre, además, Mycin era capaz de "razonar" el proceso siguiente que llevaba a los diagnósticos, permitiendo así recetar medicamentos a cada paciente según fuera su estatura, peso, etc.

En Argentina desarrollaron el sistema Huella Software basándose en sistemas expertos el cual mejora la solución para la gestión productiva de bovinos de carne. Este sistema surgió por las carencias y experiencias de productores y veterinarios dedicados a ésta actividad, fue desarrollado en conjunto con la facultad de ciencias veterinarias de la Universidad Nacional del Centro de La Provincia de Buenos Aires (Tandil.Bs.As). Este es un software comercializable disponible en dos versiones, Productores y Veterinarios, de los cuales registra todas las actividades de Cría, Invernada (CebaFeed lot), Ciclo completo, Cabañas.

En Trujillo, Perú, Pedro Nelson Shiguihara Juárez y Jorge Carlos Valverde Rebaza, construyeron SEDFE un Sistema Experto para él para el diagnóstico de las plagas y enfermedades de los cultivos de Espárrago usando Redes Bayesianas, desarrollado en la Universidad Nacional de Trujillo, Escuela de Informática.

En México en la Universidad Nacional Autónoma de México (UNAM), se participó que ya se han realizado los sistemas inteligencia artificial como son: jitomate, limón, durazno, fresa, aguacate y guayaba, estos proyectos han sido liderados por el Dr. Nicolás Kemper Valderde, investigador de y director del proyecto "Sistema Experto para la Predicción de Plagas y Enfermedades en Frutas y Hortalizas del Estado de Michoacán".

Y llegando a Colombia en el año 2003 se desarrolló un S.E. para la Toma de Decisiones de Habilidad y Reparabilidad en edificios después de un sismo que tuvo como epicentro la ciudad de Manizales, después de esto la Asociación de Ingeniería Sísmica en la Oficina Municipal para la Prevención y atención de Desastres de esta ciudad y por colaboración de Marta Liliana Carreño, Omar Darío Cardona, Ana Campos García, los cuales propusieron una herramienta de inteligencia computacional que correspondería a una aplicación desarrollada en Visual Basic, la cual utilizaría un modelo de evaluación basado en redes neuronales y lógica difusa. Este programa de computador serviría de apoyo para la valoración del daño en edificaciones afectadas por sismos, además orientar la identificación de las acciones de protección que debían realizarse en cada caso.

En el 2006 se desarrolló un S.E. de predicción de cáncer prostático a través de muestras de 
Harold Bula, Sistema Experto Berenjena

sangre por examen de antígeno prostático específico. Este trabajo fue desarrollado por el ingeniero Andrés Corredor de la Universidad El Bosque en Bogotá (Colombia). En el 2007 en la Universidad del Bosque los estudiantes Andrés Felipe Bohórquez Rodríguez y Pedro Pablo Gutiérrez Valencia, desarrollaron un SE para la clasificación de animales partiendo de sus características taxonómicas, para optar el título de Ingeniero de Sistemas cuyo principal objetivo fue Implementar el Shell de un Sistema Experto para la sistematización de claves taxonómicas para la clasificación de animales.

\section{MATERIALES Y MÉTODOS}

La elaboración del presente trabajo se llevó a cabo en la Universidad de Córdoba, Colombia.

El estudio de este estuvo conformado por cinco fases independientes pero profundamente relacionadas, que fueron desarrolladas por los autores con ayuda del comité asesor.

La primera fase fue una investigación previa que consistió en recolectar información concerniente a la construcción de un sistema experto y en hacer un estudio detallado de las herramientas o lenguajes de programación utilizados para la construcción de estos, con la intención de elegir la herramienta más apropiada para dar solución a la problemática.

La segunda fase fue una planeación, la cual tenía como objetivo producir un documento estructurado y organizado que permitiera el desarrollo del SE. Para ello se establecieron procesos tales como: Estudio de la factibilidad, determinación de las Tareas del S.E., definición de los requisitos de alto nivel.

La tercera fase fue definir la base de conocimiento sobre la cual se tomarían las decisiones que permitieran diagnosticar las plagas y enfermedades en los cultivos de berenjena mediante su sintomatología, para esto lo primero que se hizo fue escoger el comité asesor, y debido a la naturaleza del trabajo, se tomó por epicentro el Departamento de Ingeniería Agronómica y Desarrollo Rural de la universidad, es decir, Ingenieros Agrónomos especialistas en los cultivos de berenjena. El conocimiento adquirido se obtuvo de revisión de literatura como libros, artículos y revistas, pero principalmente de entrevistas personales con los especialistas en cultivos de berenjena.

La cuarta fase fue la de codificación y verificación, esta fase consistió en diseñar las reglas de inferencia que se tendrían en cuenta en la base del conocimiento, concerniente al diagnóstico de las plagas y enfermedades en los cultivos de berenjena mediante su sintomatología. De otro lado en esta fase también se diseñó la arquitectura del sistema experto para el diagnóstico de las plagas y enfermedades en los cultivos de berenjena mediante su sintomatología, para integrar todos los módulos del sistema y permitir a los usuarios la interacción con el sistema mediante una interfaz para la toma de decisiones.

La quinta y última fase consistió en evaluar el sistema, esto se logró mediante la realización de una prueba detallada del sistema, sin errores de compilación, para así poder construir el manual del usuario.

\section{RESULTADOS Y DISCUSIÓN}

Para resolver cualquier problema, de diversa índole lo primero que se debe hacer es definir el área o el dominio del problema a resolver; esta proposición es válida tanto en el campo de la inteligencia artificial (AI) como en la programación convencional. Sin embargo se han creado paradigmas y conceptos dogmáticos acerca de la Al como las afirmaciones: "Si todavía no ha sido resuelto es un problema de la Al"; otra afirmación que se oye frecuentemente es "la Al está haciendo que las computadoras actúen como en las películas.". Esta clase de concepción mental fue muy fuerte durante los años sesenta, cuando la Al se encontraba en su fase de investigación, sin embargo hoy día eso ha cambiado y la $\mathrm{Al}$ resuelve problemas reales y tiene mucha aplicabilidad comercial.

El profesor Edward Feigenbaum de la Universidad de Stanford, pionero en la 
tecnología de los sistemas expertos, los ha definido "como un programa de computación inteligente que usa el conocimiento y los procedimientos de inferencia para resolver problemas que son lo suficientemente difíciles como para requerir significativa experiencia humana para su solución [5]. Es decir, que un SE es un sistema computacional que tiene la destreza de tomar decisiones como un especialista humano. Los sistemas expertos se desprenden de la $\mathrm{Al}$ la cual se desarrolla empleando el conocimiento de un área determinada para dar solución a problemas como un especialista humano. El cual es un individuo que posee la experiencia desarrollada en un área afín. Como se sabe, el especialista humano, posee conocimientos 0 destrezas especiales de las cuales muchas personas no disponen. El conocimiento que necesite utilizar un sistema experto se obtiene mediante consultas de dichos conocimientos los cuales se encuentran disponibles en libros, revistas, artículos, y con personas capacitadas. En los S.E. los usuarios de este aportan las variables o parámetros para que así el sistema les dé una respuesta o solución. En su interior, el sistema experto incluye dos componentes principales. $\mathrm{La}$ base de conocimiento contiene el conocimiento que le permite al mecanismo de inferencia sacar conclusiones; estas son las respuestas del sistema experto a la consulta especializada del usuario.

Así la estructura de un sistema experto se encuentra compuesta por tres grandes módulos como son la base del conocimiento y el motor de inferencia y la interfaz de usuario.

Base de conocimientos. Es la porción del S.E. en donde se encuentra contenido el conjunto de conocimientos del dominio. Para que pueda estar contenido hay que adquirir el conocimiento del experto o el especialista y codificarlo en la base de conocimientos, de la manera más clara. Una forma común de hacer la representación de dicho conocimiento en un S.E. son las reglas. Las reglas son estructuras condicionales que relaciona un antecedente para lógicamente llegar al consecuente.

Motor de inferencia. El S.E. modela el proceso de razonamiento humano con un módulo conocido como el motor de inferencia. El cual trabaja con la información contenida en la base de conocimientos [6].

Interfaz de usuario. La interacción entre un S.E. y un usuario se realiza en lenguaje natural. También es altamente interactiva y sigue el patrón de la conversación entre seres humanos. Para conducir este proceso de manera aceptable para el usuario es especialmente importante el diseño de la interfaz de usuario. Un requerimiento básico de la interfaz es la habilidad de hacer preguntas. Para obtener información fiable del usuario hay que poner especial cuidado en el diseño de las cuestiones. Esto puede requerir diseñar el interfaz usando menús o gráficos.

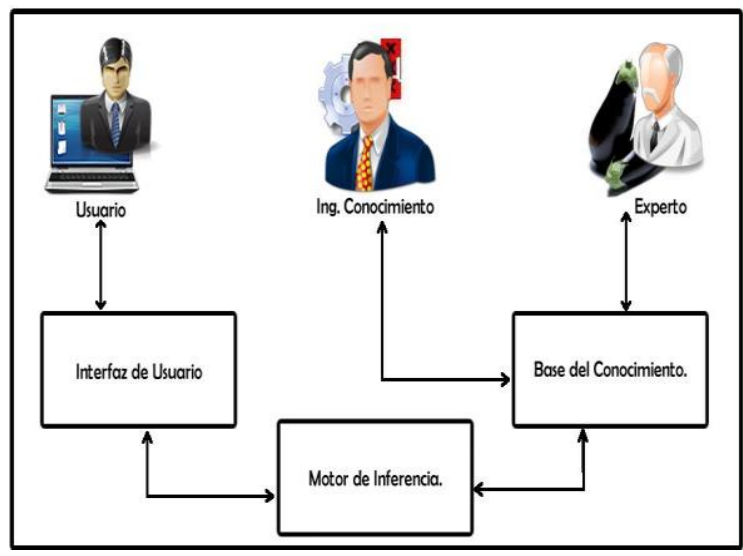

Figura 1. Estructura del sistema experto.

El primer paso para la construcción de un sistema experto, es hacer la Base del conocimiento; que consiste en entrevistas con el experto en el tema en las cuales este debe proporcionar la información necesaria al ingeniero del conocimiento, éste último a su vez selecciona, retroalimenta y de ser necesario complementa esta información que se adquieres del experto, para luego pasar a la segunda etapa se diseñan las reglas de inferencia que manejara el motor de inferencia, lo cual no es más que el comportamiento que va a tener el sistema experto con base a una entrada dada por el usuario, por medio de la interfaz de usuario; dependiendo de la información contenida en la base del conocimiento. 
La base de conocimiento para el sistema SEFI se construyó después de varias reuniones con el experto, investigando libros, artículos y revistas, después se hizo un estudio detallado de toda esa información recolectada y se escogió la información realmente relevante para el sistema, adquiriendo así el conocimiento que debía ser establecido o codificado en la base del conocimiento.

La base del conocimiento fue dividida en dos grandes módulos, el de plagas y el de enfermedades.

Este primer módulo se encuentra fraccionado en 5 secciones, que contienen las características de los tallos, las hojas, los frutos, el follaje y la planta en general que se deben encontrar en los cultivos de berenjena en caso de que estén siendo atacados por alguna plaga en especial, para poder identificar esta plaga es necesario que el usuario en cada una de las secciones del módulo escoja las características que presenta el cultivo, una sexta sección que contiene este módulo y la cual es obligatoria y de suma importancia para que el sistema pueda arrojar una diagnóstico contundente es la estación climita, al haber llenado el formulario y pulsar el botón evaluar el sistema arrojara un diagnóstico de la plaga que se encuentra afectando el cultivo, una descripción de esta y el debido tratamiento que se le debe dar a esta, el cual va a depender de la estación escogida por el usuario previamente.

El segundo módulo también se encuentra fraccionado en 5 secciones que contienen las características de los semilleros, los tallos, las hojas, los frutos y la planta en general que se deben encontrar en los cultivos de berenjena en caso de que estén siendo atacados por alguna enfermedad en especial, para poder identificar esta enfermedad es necesario que el usuario en cada una de las secciones del módulo escoja las características que presenta parametrizar todas las preguntas de la lista de chequeo desde P1 hasta P31 de acuerdo al orden asignado junto con una variable ' $Y$ ' a la que se le asignara el resultado del diagnóstico, luego mediante el condicional si, a cada el cultivo, una sexta sección que contiene este módulo y la cual es obligatoria y de suma importancia para que el sistema pueda arrojar una diagnóstico contundente es la estación climita, al haber llenado el formulario y pulsar el botón evaluar el sistema arrojara un diagnóstico de la enfermedad que se encuentra afectando el cultivo, una descripción de esta y el debido tratamiento que se le debe dar a esta, el cual va a depender de la estación escogida por el usuario previamente.

El diseño de las reglas que se utilizarían en el motor de inferencia para el sistema SEFI fueron construidas después de tener toda la información de la base del conocimiento.

El sistema experto para diagnóstico de plagas y enfermedades en cultivos de berenjena parte de una lista de chequeo, que se formuló basándose en la información proporcionada por el experto, donde el usuario al escoger una posible opción de las que se encuentran en las fracciones de cada módulo, realmente responde un "si" o "no", y esta respuesta es analizada por el motor de inferencia, mediante unas reglas donde se evalúa la inferencia y si ésta es correcta entonces el diagnóstico también debe serlo. Para obtener los diagnósticos, se utilizaron dos tipos de reglas o inferencias.

La primera regla corresponde al diagnóstico que arrojara el sistema dependiendo de la entrada que digite el usuario en las 31 preguntas $u$ opciones que se encuentran en la lista de chequeo del módulo de plagas, después que se captura el valor de estas el sistema analiza y compara en el motor de inferencia si estas capturas coincide con las reglas que se alojan en este, para que el sistema pueda lanzar un diagnóstico.

En el módulo de plagas la sintaxis de esta primera regla consiste en parámetro se le asigna una variable "sí" o "no", y dependiendo de los valores que tenga cada pregunta se arrojara el diagnóstico o nombre de la plaga que será guardado en la variable ' $Y$ '. Así: 
Harold Bula, Sistema Experto Berenjena

plagas $(P 1, P 2, P 3, P 4, P 5, P 6, P 7, P 8, P 9, P 10, P 11, P 12, P 13, P 14, P 15, P 16, P 17, P 18, P 19, P 20, P 21, P 22, P 23$,

$P 24, P 25, P 26, P 27, P 28, P 29, P 30, P 31, Y):-P 1=' n 0^{\prime}, P 2=' n 0^{\prime}, P 3=' n 0^{\prime}, P 4=' n 0^{\prime}, P 5=' n 0^{\prime}, P 6=' s i i^{\prime}, P 7='$

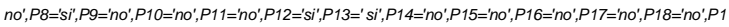

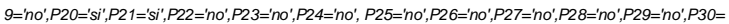

'no',P31='no', Y='nombreplaga'

En el módulo de plaga se hace uso nueve veces de esta sentencia, ya que el sistema diagnóstica 9 plagas y debe hacer una inferencia por cada plaga a diagnosticar.

La segunda regla corresponde al control que arrojara el sistema dependiendo de la entrada que digite el usuario en la sexta sección de preguntas $u$ opciones que se encuentran en la lista de chequeo del módulo de plagas, en la cual es obligación escoger una de las dos opciones para que después el valor capturado sea analizado y comparado con el que se encuentra en el motor de inferencia, si estas capturas coincide con las inferencias que se alojan en este, para que el sistema pueda lanzar un control.

En el módulo de plagas la sintaxis de la segunda regla consiste en parametrizar tres variables $L, P, Y$, donde, $L$ captura el valor de la estación climática, la cual trae asignado un "seca" o "lluviosa", P trae el diagnóstico de la plaga y por ultimo $Y$ guardara el control que lanzara el sistema, luego mediante el condicional si, se comparan estas variables y dependiendo de la estación climática el sistema arrojara un control. Así:

control $(L, P, Y):-L=$ 'seca', $P=$ 'acaro', $Y=$ 'plaga1'. control $(L, P, Y):-L=$ 'seca', $P=$ 'acaro', $Y=$ 'plaga2'.

En el módulo de plaga se hace uso dieciocho veces de esta sentencia, ya que el sistema diagnóstica 9 plagas y debe hacer dos inferencia por cada plaga ya que se debe tener en cuenta la estación climática, para que el sistema arroje un control.

En el módulo de enfermedades la sintaxis de esta primera regla consiste en parametrizar todas las preguntas de la lista de chequeo desde P1 hasta P20 de acuerdo al orden asignado junto con una variable ' $Y$ ' a la que se le asignara el resultado del diagnóstico, luego mediante el condicional si, a cada parámetro se le asigna una variable "sí" o "no", y dependiendo de los valores que tenga cada pregunta se arrojara el diagnóstico o nombre de la enfermedad que será guardado en la variable ' $Y$ '. Así:

enfermedad $(P 1, P 2, P 3, P 4, P 5, P 6, P 7, P 8, P 9, P 10, P 1$

1,P12,P13,P14,P15,P16,P17,P18,P19,P20,Y):P1='s

i',P2='si',P3='si',P4='no',P5='no',P6='no',P7='no',P8

$=' n o^{\prime}, P 9={ }^{\prime} n o^{\prime}, P 10=' n o$ ',P11='no',P12='no',P13='no',P

14='no',P15='no',P16='no',P17='no',P18='no',P19='n

o', $P 20=' n o$ ', $Y=$ 'nombreenfermedad'.

En el módulo de enfermedades hace uso ocho veces de esta sentencia, ya que el sistema diagnóstica ocho enfermedades y debe hacer una inferencia por cada enfermedad a diagnosticar.

En el módulo de enfermedades la sintaxis de la segunda regla consiste en parametrizar tres variables $L, P, Y$, donde, $L$ captura el valor de la estación climática, la cual trae asignado un "seca" o "lluviosa", P trae el diagnóstico de la enfermedad y por ultimo Y guardara el control que lanzara el sistema, luego mediante el condicional si, se comparan estas variables y dependiendo de la estación climática el sistema arrojara un control. Así:

controlenf $(L, P, Y)$ :-

$L=$ 'seca', $P=$ 'damping', $Y=$ 'enfermedad1'.

controlenf $(L, P, Y)$ :-

$L=' / l u v i o s a ', P=$ 'damping', $Y=$ enfermedad2'.

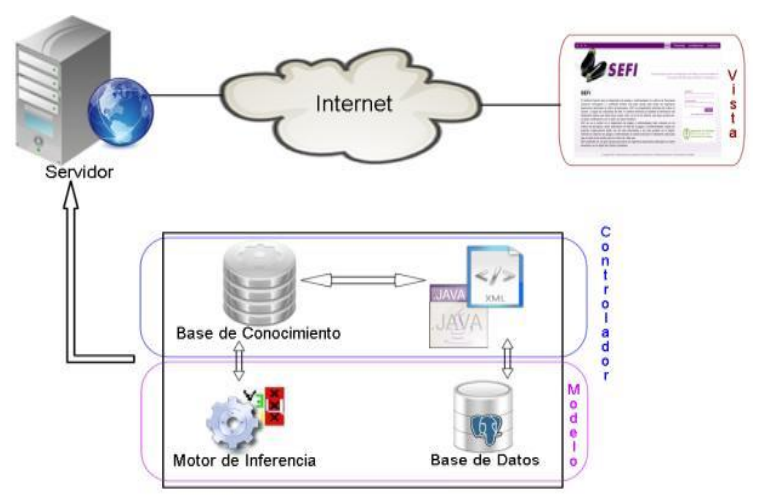

Figura 2. Arquitectura del sistema experto. 


\section{CONCLUSIONES}

Por otra parte la arquitectura del sistema experto SEFI se desarrolló en un ambiente Web en tres capas: modelo, controlador y vista.

En la capa de modelo se encuentra el motor de inferencia, el cual se encarga de analizar los hechos que se encuentran en la base de conocimiento, según sus reglas y generar una respuesta, además se encuentra PostgreSQL un motor de bases de datos rápido, robusto y multi-plataforma, por lo cual es una buena opción para aplicaciones basadas en la web.

La capa controlador se encuentra administrada por clases Java, ya que este es un lenguaje multiplataforma, rápido, de uso sencillo, programación orientada a objetos y se puede integra con varios gestores de bases de datos entre los que se encuentra PostgreSQL, las clases javas se encarga de controlar tránsito entre la capa de vista y modelo.

La capa vista se encarga de recibir datos de la capa modelo y mostrarlos al usuario a través de la interfaz a la cual podrá acceder mediante internet, que en su mayoría tiene contenido JSP y XML, debido a que son lenguajes estándares para la mayoría de los navegadores con que se cuenta hoy día.

La estructura del sistema experto está compuesta por tres actores y por tres partes; que se interrelacionan entre sí.

\section{EVALUACIÓN DEL SISTEMA}

La evaluación del sistema se llevó a cabo con la ayuda de expertos colaboradores, el sistema fue probado por Ingenieros agrónomos y estudiantes de agronomía de VIII a X semestre los cuales contaban con las bases fundamentales para dar criterios sobre los resultados obtenidos, así estos tuvieron en cuenta en la prueba, facilidad en el uso del sistema, numero de aciertos, tiempo de respuesta del sistema. Después de que los expertos dieran el visto bueno del sistema se procedió a realizar el manual del usuario.
Al desarrollar el sistema experto para el diagnóstico de plagas y enfermedades en los cultivos de berenjena se utilizaron varias tecnologías, Prolog, JAVA ${ }^{\mathrm{TM}}, \mathrm{Xml}, \mathrm{API}$ de java como Jfreechart, Apache Tomcat, Postgres, NetBeans que permitió el desarrollo del sistema para el lenguaje Swi-Prolog y por medio de estos se lograron satisfacer las exigencias del desarrollo de la investigación.

El Sistema se provee como una gran herramienta educativa dentro del proceso de enseñanza a personas interesadas en los cultivos de berenjena.

El sistema implementado ofrece una velocidad de respuesta muy alta, según lo obtenido en la etapa de pruebas. Esto corrobora la teoría base del presente proyecto, de que el sistema experto tomara decisiones mucho más rápidas y eficientes de lo que lo haría un experto humano.

\section{REFERENCIAS}

[1]. Aramendiz, H., Cardona, C., Jarma, A. y Espitia, M., (2008). El cultivo de la berenjena (Solanum melongena L.). Montería: Produmedios.

[2]. http://www.ncbi.nlm.nih.gov/entrez/quer y.fcgi?db=pubmed\&cmd=Retrieve\&dopt $=$ AbstractPlus\&list ds $=10520680 \&$ query_hl $=13 \&$ itool=pub med_docsum.

[3]. http://protic.org/proyectos.shtml?x=201 92285

[4]. Feigenbaum, E. (2001) Sistemas Expertos Principios y Programación, Madrid: Internacional Thomson Editores. p. 1-2.

[5]. http://informaticosistemas.wordpress.co m/2011/03/23/arquitectura-basica-de-lossistemas-expertos/ 
[6]. CULTIVOS, P. (2010). Periodo de interferencia de arvenses en el cultivo de berenjena (Solanum melongena I.). Agronomía Colombiana, 28(1), 8188.

[7]. Cisneros, F. L. M. (1991). Sistema Experto para Diagnóstico de Plagas Insectiles de Maíz (Zea mays L.) en Centro América1. AGRONOMÍA MESOAMERICANA, 2, 80-88.

[8]. Sánchez L, C. (1993). Diseño de un sistema experto para la identificación de enfermedades en el cultivo del arroz Oryza sativa L. en Colombia.--p. 134144.

[9]. Lopez de Rojas, I., Zacarias, M. D., \& Comerma, J. A. (1987). Avances en la construcción de un sistema experto para suelos ácidos de Venezuela.[Progress in the development of an expert system for acid soils of Venezuela]. In 10. Congreso Latinoamericana de la Ciencia del Suelo y 9. Congreso
Venezolano de la Ciencia del Suelo. Maracaibo (Venezuela). 14-21 Jun 1987.

[10]. Barragán, Á., Cajamarca, N., Salazar, D., \& Indira, N. (2012). Sistema Experto para Decisiones de Riego en Cultivos de Cacao.

[11]. Villafuerte Zea, L. E. (1998). Sistemas expertos como herramienta para toma de decisiones de manejo en sistemas silvopastoriles del trópico húmedo bajo de Costa Rica (Doctoral dissertation, Tesis MSc. Turrialba, Costa Rica, CATIE).

[12]. Saenz, T. C., Vargas, G. A., \& Torres, R. M. (2000). Sistema computarizado experto en diagnóstico nutrimental en naranjo. Publicado en Internet http:/MWW.

chapíngomx/terra/contenido/8/2/a1t173 -178. pdf [Fecha de acceso:

Noviembre 2005]. 